\title{
FINGERPRINTS IMAGE COMPRESSION BY WAVE ATOMS
}

\author{
Mustapha Delassi and Amina Serir. \\ LTIR, Faculté d'électronique et d'informatique, USTHB \\ BP 32 EI Alia, bab ezzouar, 16111 Alger, Algerie
}

\begin{abstract}
The fingerprint images compression based on geometric transformed presents important research topic, these last year's many transforms have been proposed to give the best representation to a particular type of image "fingerprint image", like classics wavelets and wave atoms. In this paper we shall present a comparative study between this transforms, in order to use them in compression. The results show that for fingerprint images, the wave atom offers better performance than the current transform based compression standard. The wave atoms transformation brings a considerable contribution on the compression of fingerprints images by achieving high values of ratios compression and PSNR, with a reduced number of coefficients. In addition, the proposed method is verified with objective and subjective testing.
\end{abstract}

\section{KEYWORDS}

Image, compression, fingerprint, wavelets, wave atoms, WSQ.

\section{INTRODUCTION}

The fundamental goal of image compression is to obtain the best possible image quality at an allocated storage capacity. For this, data compression is one of the major challenges that are used in the majority of digital applications and specifically in the field of biometric "fingerprint", which presents the centre of interest in our work. The overall process of image compression through a series of steps: transformation, quantization and coding. The diversity at the steps led to the birth of different compression standards according to the desired application.

In image compression, it is important to get the ability of representing in a very simple way the data or the information with the minimum possible elements with allowing a loss of information, for this, the transformation has duel contribution, it decorrelates the image components and allows identifying the redundancy. Second it offers a high level of compactness of the energy in the spatial frequency domain. There have been several transforms used in data compression, Discrete Fourier Transform (DFT) and the DCT (Discrete cosines transform), DWT (Discrete wavelets transform) for images compression.

Wavelets have been widely used in image processing, such as denoising and image compression [1]. The success of wavelets with the JPEG2000 standard, and the DCT with the JPEG standard was great, but its performance is limited to a certain type of images, this is why some other standard dedicated to compression of fingerprint images are appeared, one of them is, the FBI

David C. Wyld et al. (Eds) : CST, ITCS, JSE, SIP, ARIA, DMS - 2014

pp. 271-278, 2014. (C) CS \& IT-CSCP 2014

DOI : $10.5121 /$ csit.2014.4125 
fingerprint image compression standard, the wavelet scalar quantization (WSQ), which is based on optimized decomposition of wavelet.

In our work we propose an algorithm for fingerprint image compression using wave atoms decomposition, compared with WSQ standard results, in order to justify the contribution that can bring wave atoms transformation for fingerprint image compression.

The remainder of this paper is divided into 4 sections. Section 2 discusses the wavelets and WSQ standard, wave atoms transform implementation details and the proposed method for fingerprint image compression is described in section 3. Experimental results are discussed in section 4 and section 5 details concluding remarks followed by acknowledgment and references.

\section{WSQ STANDARD}

In 1993, the FBI (Federal Bureau of Investigation) has developed a standard for compressing fingerprint images and reconstruction based on wavelet transforms, the fingerprint image is decomposed using 2D DWT, the wavelets used are the biorthogonal 9/7 discrete wavelet transform (DWT), by decomposing the image into four sub-bands of lower size in every level. The structure of the tree decomposition can be determined by applying different tests on several reference images. The tests concluded that the best tree consists of 64 sub bands [2]. These subbands are further decomposed in to sets which grouped the same levels of decomposition, then quantized and coded. The quantization used for each sub-bands is, the uniform scalar quantization with dead zone, and then encoded using Huffman algorithm and RLE (Run Length Encoding). The WSQ compression technique can compress fingerprint images with compression ratio ranging from 10 to 1 and 20 to 1 [2]. However, despite that the wavelet transform is effective for the detection of isotropic structures; it's not optimal for the analysis of anisotropic objects in the image (i.e., lines, contours), because they fail to follow the direction of this edge. To this effect, new multiscale geometric transforms so-called second generation have been developed, such as ridgelets [3], contourlets [4], curvelets [5], and more recently proposed, the wave atoms, which all incorporate the notion of directionality.

\section{WAVE ATOMS}

Wave atoms are a recent addition to the collection of mathematical transforms for harmonic computational analysis. Wave atoms are a variant of wavelet packets, they have a high frequency localization that cannot be achieved using a filter bank based on wavelet packets and curvelet Gabor atoms. Wave atoms precisely interpolate between Gabor atoms [6] (constant support) and directional wavelets [7] (wavelength $\sim$ diameter) in the sense that the period of oscillations of each wave packet (wavelength) is related to the size of essential support by the parabolic scaling i.e. wavelength $\sim$ (diameter)2.

Different transforms based on wavelet packet, need to be represented as 'phase-space tiling', there are two different parameters $\alpha$, which represent whether the multi-scale decomposition is , and $\beta$ directional capacity if it is isotropic, Wavelets (including Multi Resolution Analysis [5], directional [8] and complex [9]) correspond to $\alpha=1 \beta=1$, for ridgelets [10] $\alpha=1, \beta=0$, Gabor transform $\alpha=1, \beta=0$ and curvelets [3] correspond to $\alpha=1, \beta=1 / 2$. Wave atoms are defined for $\alpha=1, \beta=1 / 2$. Figure 1 illustrates this classification [4]. 


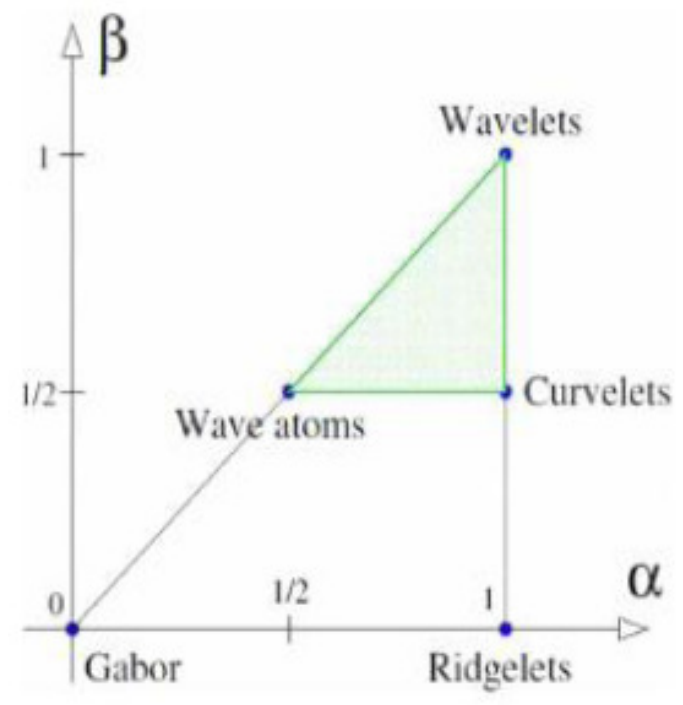

Figure 1. Diagram of $(\alpha, \beta)$ [Hadd 2009]

\subsection{D discrete wave atoms}

Wave atoms are tensor products of a special type of 1D wave packets. $\psi_{m, n}^{j}(x)$ is a onedimensional family of real-valued wave packets, where $j \geq 0, m \geq 0$ and $n \in \mathrm{Z}$, cantered in frequency around $\pm \omega \mathrm{j}, \mathrm{m}=2 \pi \mathrm{Jm}$, with $C_{1^{2}}{ }^{j} \leq m \leq C_{2} 2^{j}$, and centred in space around $\mathrm{xj}, \mathrm{n}=2-\mathrm{jn}$. Onedimensional version of the parabolic scaling states that the support of $\hat{\psi}_{m, n}^{j}(\omega)$ is of length $\mathrm{O}(2 \mathrm{j})$ while $\omega \mathrm{j}, \mathrm{m}=\mathrm{O}(22 \mathrm{j})$. Filter bank-based wavelet packets is considered as a potential definition of an orthonormal basis satisfying these localization properties. The wavelet packet tree, defining the partitioning of the frequency axis in $1 \mathrm{D}$, can be chosen to have depth $\mathrm{j}$ when the frequency is $22 \mathrm{j}$. However, there is a problem associated with standard wavelet packets, that the direction in which they meet the frequency localization is rather weak [9].

Dyadic dilates and translates of $\hat{\omega}_{m}^{0}$ on the frequency axes are combined and bases function, written as:

$$
\psi_{m, n}^{j}(x)=\psi_{m}^{j}\left(x-2^{-j} n\right)=2^{j / 2} \psi_{m}^{0}\left(2^{j} x-n\right)
$$

The coefficients cj,m,n, for each wave number $\omega \mathrm{j}, \mathrm{m}, \mathrm{n}$, are obtained as a decimated convolution at scale 2-j.

$$
c_{j, m, n}=\int \psi_{m}^{j}\left(x-2^{-j} n\right) u(x) d x
$$

By Plancherel's theorem,

$$
c_{j, m, n}=\int e^{i 2^{-j} \overline{\wedge^{j}}} \hat{\psi_{m}(\omega) u(\omega) d \omega}
$$

Assuming that the function $\mathrm{u}$ is accurately discretized $\mathrm{xk}=\mathrm{kh}, \mathrm{h}=1 / \mathrm{N}, \mathrm{k}=1 \ldots . \mathrm{N}$, then up to some small truncation error: 


$$
c_{j, m, n}^{D}=\sum_{k=2 \pi(-N / 2+1 \cdot 1: N / 2)} e^{i 2^{-j} \overline{\wedge^{j}} \hat{\psi_{m}(k) \hat{u}(k)}}
$$

This equation makes sense for couples $(\mathrm{j}, \mathrm{m})$ for which the support of $\hat{\psi}_{m}^{j}(k)$ lies entirely inside the interval $[-\pi n, \pi n]$, so we may write $k \in 2 \pi \mathrm{Z}$.

\subsubsection{D discrete wave atoms}

A two-dimensional orthonormal basis function with 4 bumps in frequency plane is formed by individually taking products of $1 \mathrm{D}$ wave packets. Mathematical formulation and implementations for $1 \mathrm{D}$ case are detailed in the previous section. $2 \mathrm{D}$ wave atoms are indexed by $\mu=(\mathrm{j}, \mathrm{m}, \mathrm{n})$, where $\mathrm{m}=(\mathrm{m} 1, \mathrm{~m} 2)$ and $\mathrm{n}=(\mathrm{n} 1, \mathrm{n} 2)$. Construction is not a simple tensor product since there is only one scale subscript $\mathrm{j}$. This is similar to the non-standard or multiresolution analysis wavelet bases where the point is to enforce same scale in both directions in order to retain an isotropic aspect ratio. Eq. (1) is modified in $2 \mathrm{D}$ as:

$$
\varphi_{\mu}^{+}\left(x_{1}, x_{2}\right)=\psi_{m_{1}}^{j}\left(x_{1}-2^{-j} n_{1}\right) \psi_{m_{2}}^{j}\left(x_{2}-2^{-j} n_{2}\right)
$$

The Fourier transform is also separable, namely:

$$
\begin{aligned}
& \hat{\varphi}_{\mu}^{+}\left(\omega_{1}, \omega_{2}\right)=\hat{\psi}_{m_{1}}^{j}\left(\omega_{1}\right) e^{-i 2^{j} n_{1} \omega_{1}} \hat{\psi}_{m_{2}}^{j}\left(\omega_{1}\right) e^{-i 2^{j} n_{2} \omega_{2}} \\
& \varphi_{\mu}^{-}\left(x_{1}, x_{2}\right)=H \psi_{m_{1}}^{j}\left(x_{1}-2^{-j} n_{1}\right) H \psi_{m_{2}}^{j}\left(x_{2}-2^{-j} n_{2}\right)
\end{aligned}
$$

The recombination between the relations (6) and (7), by dual orthonormal basis give:

$$
\varphi_{\mu}^{(1)}=\frac{\varphi_{\mu}^{+}+\varphi_{\mu}^{-}}{2}, \quad \varphi_{\mu}^{(2)}=\frac{\varphi_{\mu}^{+}-\varphi_{\mu}^{-}}{2}
$$

provides basis functions with two bumps in the frequency plane, symmetric with respect to the origin, hence directional wave packets (oscillating in one single direction). Together, $\varphi_{\mu}^{(1)}$ and $\varphi_{\mu}^{(2)}$, form the wave atom frame and may be denoted jointly as $\varphi_{\mu}$. The price to pay in considering both $\varphi_{\mu}^{(1)}$ and $\varphi_{\mu}^{(2)}$, is an increase of a factor 2 in the redundancy.

Wave atom algorithm is based on the apparent generalization of the 1D wrapping strategy to two dimensions and its complexity is $\mathrm{O}(\mathrm{N} 2 \log \mathrm{N})$.

Figure 2 represents the wave atom tiling of the special frequency plane. The size of the squares doubles when the scale $\mathrm{j}$ increases by 1 . At a given scale $\mathrm{j}$, squares are indexed by $\mathrm{m} 1, \mathrm{~m} 2$ starting from zero near the axes. 


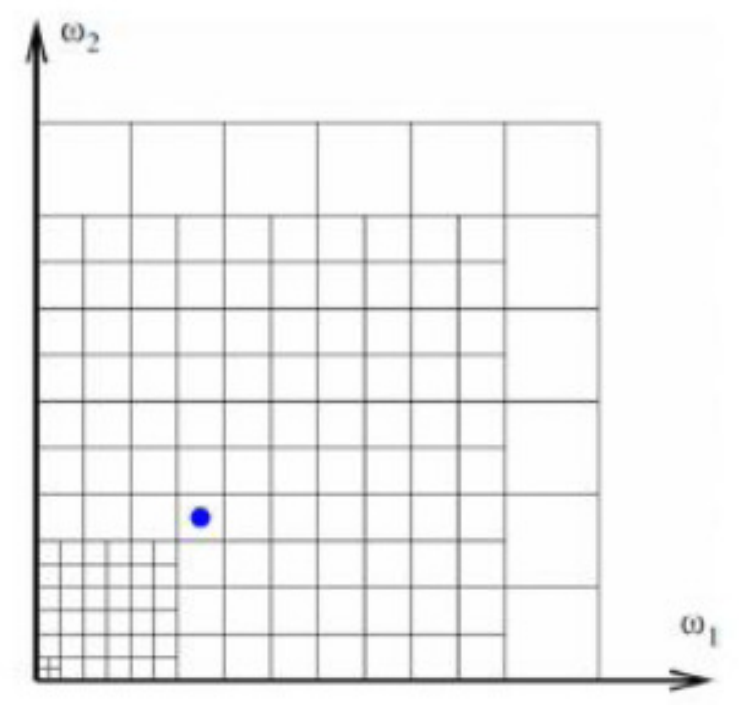

Figure 2. wave atom tiling of the frequency plane

\section{RESULUTS AND DISCUTION}

\subsection{Transforms studies}

Several fingerprint images compression standards was used in order to reduce their size, quoting JPEG with CDT transforms, WSQ, and JPEG 2000 with DWT transforms, wavelet transform has brought a significant contribution, but for the feature of the fingerprint images, these transformations have not given a good compression results, for this, other transforms was used to better characterize the fingerprints images.

Measures such as MSE (mean squared error) or PSNR (peak signal to noise ratio), correspond to the numerical analysis of pixel values before and after compression, these values are very general and do not always reflect the quality of the reconstructed image. For an image I of size $\mathrm{n} \times \mathrm{m}$ pixels, we define:

$$
\begin{gathered}
M S E=\frac{1}{n \times m} \sum_{i=1}^{n} \sum_{j=1}^{m}(I-\hat{I})^{2} \\
P S N R=10 \log _{10} \frac{(256-1)^{\wedge}}{M S E} \text { en } d B
\end{gathered}
$$

$\hat{I}$ represents the compressed image.

The purpose of each transformation is to concentrate the information on few coefficients, for this we are going to precede tests to reconstruct the image with a portion of the coefficients.

In this paper we propose algorithms for fingerprint images compression based on wavelets and wave Atoms transform. First, we apply the different transforms on the original image in order to obtain the energy maximization on a reduced number of coefficients; following the low coefficients will be set to zero by a threshold. The image is reconstructed in this case only by the 
most significant coefficients, and calculates the PSNR for determining the quality of the reconstructed image.

Tables 1 and 2 present a comparative study between different transformations, based on wavelets transforms and wave atoms, and their effects on reducing the number of coefficients required for image reconstruction.

Table 1. PSNR's results with wavelets transforms

\begin{tabular}{|c|c|c|c|c|c|c|c|}
\hline $\begin{array}{l}\text { Coefficients } \\
\text { selected (\%) }\end{array}$ & 10 & 20 & 30 & 50 & 80 & 90 & 100 \\
\hline wavelets & 33.95 & 34.05 & 34.25 & 34.33 & 34.39 & 34.40 & 34.44 \\
\hline W P & 34.45 & 34.58 & 34.66 & 34.80 & 35.20 & 35.32 & 35.40 \\
\hline WSQ & 34.08 & 34.15 & 34.36 & 34.42 & 34.73 & 34.78 & 34.85 \\
\hline
\end{tabular}

From the Tables 1 and 2 we note that the PSNR increases when the percentage of coefficients selected for image reconstruction increase. This applies to all transformations, by varying the threshold value. However, this increase differs from decomposition to another, and in the same context, we see that it is not a large scale for wavelet-based transformations. While with the transformation in wave atom, the PSNR is growing strongly and the values are higher compared to other processing while the number of coefficients used for reconstruction is much less important.

Table 2. PSNR's results with wave atoms transforms

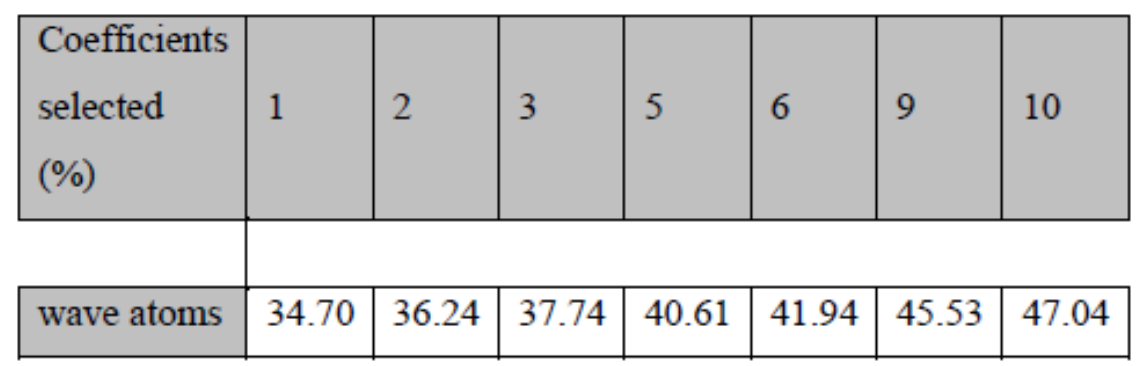

We conclude that the transformation wave Atoms can better focus the information useful for the image reconstruction, on few coefficients, this give more importance to use the wave atoms transform for the compression of fingerprints images.

\subsection{Compression algorithms}

In this section, we realized the several compression schemes based on transformations wavelets and wave atoms.

All these methods are based on the same compression scheme: we start by the transformation, after the quantization and finally coding. So the difference between these schemes lies in the choice of the transformation and the strategy of quantification. For the quantization, we adopt the uniform scalar quantization with dead zone for the WSQ algorithm compression, while for the 
compression algorithm based on wave atoms transform we chose non-uniform scalar quantization, these quantization are followed by Huffman and RLE (Run length coding) coding.

Table 3. Comparative results between algorithms

\begin{tabular}{|l|l|l|l|}
\hline Algorithms & RATE & $\begin{array}{l}\text { PSNR } \\
(\mathrm{dB})\end{array}$ & $\begin{array}{l}\text { Visual } \\
\text { assessment }\end{array}$ \\
\hline wavelets & 6,17 & 31,20 & Poor \\
\hline WSQ & 5,75 & 31,36 & Poor \\
\hline $\begin{array}{l}\text { Wave } \\
\text { atoms }\end{array}$ & 18.00 & 35.04 & Very good \\
\hline
\end{tabular}

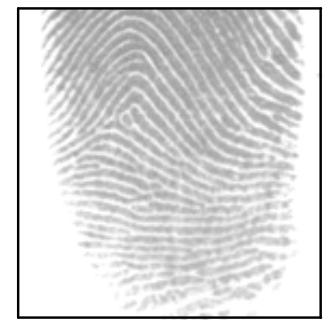

(a)

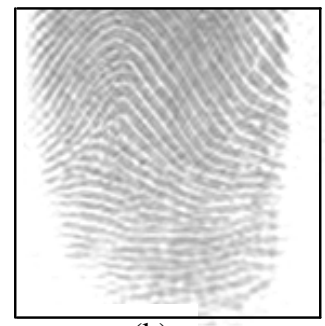

(b)

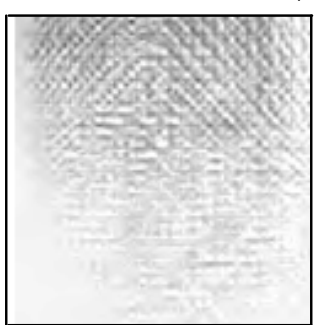

(c)

Figure 3. (a) Original image (b) Compressed image with wave atoms algorithm, (c) Compressed image with WSQ algorithm

As shown in the table 3 we can say that the wave atoms algorithm gives the best results PSNR with high compression rate.

Figure 3 represents a sample fingerprint image compressed and reconstructed by wave atoms and WSQ algorithms; we can see that wave atoms algorithm provides excellent results for the image reconstruction.

To appreciate the compression results, we could apply minutiae detection. Figure 4 illustrates the detected minutiae for original and decompressed images, we can notice that the wave atoms prevue the local structures. 

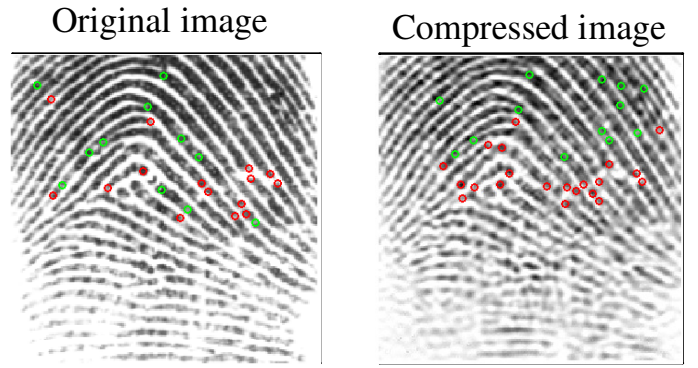

Figure 4. Minutiae detection on original and compressed image by wave atoms

The obtained results show that wave atoms transform is more appropriate to fingerprints images compression than wavelet transform

\section{CONCLUSION}

Compression of fingerprint images based on wave atoms provides better results, it can improved the PSNR with high RATE compared to WSQ fingerprint standard compression, we have also shown that wavelets are not all time appropriate to different type of images and on of them the images with curvatures and lines.

\section{ACKNOWLEDGEMENTS}

Thank's everyone

\section{REFERENCES}

[1] S. G. Mallat. “A Wavelet Tour of Signal Processing”. 2nd Edition,San Diego : Academic Press, 1999.

[2] Zehira Haddad, Azeddine Beghdadi, Amina Serir, Anissa Mokraoui, "A new fingerprint image compression based on wave atoms transform”, IEEE Xplore, 2009.

[3] M.N. Do and M. Vetterli, "The finite ridgelet transform for image representation", IEEE Trans Image Processing, vol. 12, no 1, pp. 16-28, 2003.

[4] M.N. Do and M. Vetterli, "The contourlet transform: An efficient directional multiresolution image representation", IEEE Trans. Image Processing, vol. 14, no 12, pp. 2091-2106, 2005.

[5] E.J. Candès, L. Demanet, D.L. Donoho and L. Ying, "Fast discrete curvelet transforms", Multiscale Model. Simul., pp. 861-899, 2005.

[6] S. Mallat, "A wavelet Tour of Signal Processing", Second Edition, Academic Press, OrlandoSanDiego, 1999.

[7] J.P. Antoine and R. Murenzi, "Two-dimensional directional wavelets and the scale-angle representation”, Sig. Process, vol. 52, pp. 259-281, 1996.

[8] L. Villemoes, "Wavelet packets with uniform time-frequency localization", in Comptes Rendus Math, vol. 335, no 10, pp. 793-796, 2002.

[9] L. Demanet and L. Ying, "Wave Atoms and Sparsity of Oscillatory Patterns", Appl. Comput. Harmon. Anal., vol. 23, no 3, pp. 368-387, 2007.

[10] L. Demanet and L. Ying, "Scattering in Flatland: Efficient Representations via Wave Atoms", in Found. of Comput. Math, 2008. 